\title{
How joint line orientation affects the amount of bone resection in mechanically aligned TKA: a retrospective clinical-radiographic correlation study
}

\section{Zhiwei Wang}

Beijing Chaoyang Hospital https://orcid.org/0000-0002-5668-075X

\section{Yang Yu}

Beijing Chaoyang Hospital

\section{Liang Wen}

Beijing Chaoyang Hospital

\section{Desi Ma}

Beijing Chaoyang Hospital

Tiebing Qu ( $\nabla$ qtb@medmail.com.cn )

The Center of Diagnosis and Treatment for Joint Disease, Beijing Charity Hospital, Capital Medical University https://orcid.org/0000-0003-2800-5404

\section{Research article}

Keywords: Joint line, Bone resection, Osteoarthritis, Alignment, Total knee arthroplasty

Posted Date: July 7th, 2020

DOl: https://doi.org/10.21203/rs.3.rs-38300/v1

License: (c) (i) This work is licensed under a Creative Commons Attribution 4.0 International License. Read Full License 


\section{Abstract \\ Background}

The discrepancy of bone resections between the medial and the lateral compartment is very common in total knee replacement (TKA) when mechanical alignment (MA) is used. The purpose of this study was to explore whether or how the joint line orientation affects the gross amount of bone resection in mechanically aligned TKA.

\section{Methods}

A total of 112 patients (137 knees) diagnosed with osteoarthritis (OA) were included. Simulated bone cuttings were conducted on the coronal view using full-length weight bearing radiographs according to the technical requirement of MA, and the feasibility of simulated osteotomy was verified by intraoperative calipered measurement. Correlation and regression analyses were conducted between the amounts of bone resection and various parameters, including hip-knee-ankle (HKA) angle, mechanical lateral distal femoral angle (mLDFA), joint line congruency angle (JLCA), and medial proximal tibial angle (MPTA). Moreover, any intraoperative bone resection adjustments if existed, were retrospectively collected using medical records query system.

\section{Results}

All knees in current case series can be artificially divided into 4 subgroups: 84 varus knees $(61.3 \%)$ with valgus femur in subgroup 1, 32 varus knees (23.4\%) with varus femur in subgroup 2. 14 valgus knees $(10.2 \%)$ with varus tibia in subgroup 3, 7 valgus knees (5.1\%) with valgus tibia in subgroup 4 . The amount of simulated bone resection had high consistency with the intraoperative measurement. MPTA and mLDFA had a positive correlation with the bone resection amount in subgroup 1 . The regression equation was Bone resection $(\mathrm{mm})=0.556 * \mathrm{mLDFA}$ (deg.) $+0.098 * \mathrm{MPTA}$ (deg.) $-39.74, \mathrm{R}^{2}=0.808$. Similarly, the intraoperative bone cutting adjustments also showed a certain relationship with mLDFA in subgroup 1.

\section{Conclusions}

Deformities of knee OA can be divided into 4 subgroups based on the alignment of extremities and the joint line orientations. When a TKA is performed in varus knee with valgus femur using conventional instrument and mechanical alignment technique, the amount of bone resection on coronal plane is linearly related to the patient's mLDFA.

\section{Background}


Restoration of knee neutral mechanical axis is considered as the alignment target in a total knee arthroplasty (TKA) when mechanical alignment technique is applied. The postoperative neutral alignment of lower extremity within a deviation of $3^{\circ}$ had been proved to distribute the contact forces evenly, so as to improve the long-term survivorship of implants (1-4). Both of femoral and tibial components should be implanted perpendicular to the mechanical axis of low extremities $(1,5)$.

According to the published literatures and our clinical experiences, when patients with knee osteoarthritis (OA) receiving TKA, the resection thicknesses of the medial and lateral condyles of the distal femur are significantly different $(6,7)$, Similarly, the resection thicknesses of the medial and lateral tibial plateau are different as well (8). As a consequence, the extension gap (EG) could differ significantly between medial and lateral compartments, even the overall EG may become narrower or wider which is the reason why the cutting blocks, either distal femoral or tibial ones, generally provide at least $+/-2 \mathrm{~mm}$ parallel pin holes for resection level adjustment in nearly all conventional TKA instruments. The imbalanced medial or lateral EG could be corrected by osteophytes removal or ligaments release, as well as sometimes a relatively narrower EG need additional distal femur recut to accommodate the implants. While a wider EG indicates undesirable bone loss, a thicker polyethylene insert or distal femoral augments might be mandatory to achieve extension stability and appropriate joint line level.

As mentioned above, EG has a high variability in actual surgical operations, especially in cases of using conventional instruments. Sometimes uneven EG is iatrogenic consequence from accidental mishandling of surgeons. The natural knee joint line inclination, however, might play an important role in such clinical scenarios. The hypothesis of current study is that the joint line inclination could affect the thickness of the primary bone resection in TKA. Various parameters of joint line orientation of OA knees were measured on anteroposterior full-length weight bearing roentgenograms. Bone cut simulations were performed to evaluate the gross bone loss in the EG. The relationship between various measurement parameters and supposed EG space were investigated to evaluate the effects of the joint line inclination on $E G$ in TKA.

\section{Patients And Methods}

\section{Patients}

Between July 2017 and July 2019, a total of consecutive 112 patients (patient demographics are shown in Table 1) diagnosed with OA receiving primary TKAs (137 knees) in our hospital were included in current study. The inclusion criteria are Grade III-IV Kellgren-Lawrence knee OA patients, with their full-length weight bearing radiograph of both lower extremities were obtained in which the projection of patella centered between the femoral condyles. The exclusion criteria included fixed subluxation or dislocation of the patella, congenital lower extremity deformity, history of injury or operation around knee joint, significant corrosion of subchondral bone or significant deformities compared with contralateral knee, $>10$ degrees of flexion contracture, and inflammatory arthritis.

\section{Roentgenographic measurements and bone cut simulation}


Acquisition of standard full-length weight bearing radiograph. In frontal plane, AP view, the full-length weight bearing radiographs were obtained under following conditions: Definium 8000 digital radiographic system (GE, MA, USA) with 1.8 meters of exposure distance, $80 \mathrm{Kv}$ of voltage, $400 \mathrm{~mA}$ of electric current and auto exposure mode was used in this study. Patients usually were instructed to stand with the feet together and patella faced forward. In case of tibial torsion, rotate the foot to orient the patella forward. Hip, knee and ankle joints were exposed separately, and full-leg weight bearing radiographs were automatically spliced and synthesized by the system.

Determination of anatomical landmarks and joint line parameters. Picture archiving and communication systems (PACS) were used for radiological measurements in this study. The determination of anatomical landmarks and various joint orientation angles referred to the method described by Paley et al(9). The center of femoral head was identified using Mose circles approach. The center of distal femur, tibial plateau and ankle joint were defined using the top of the femoral notch, the center of the tibial spines and the mid-width of the talus, respectively. The mechanical axis of femur and tibia were subsequently determined by connecting the above anatomical points. The distal femoral joint line was defined as the line tangential to medial and lateral femoral condyles, the proximal tibial joint line was similarly defined as the line tangential to the concave aspect of the tibial plateau subchondral line.

Measurement of joint orientation parameters. Hip-knee-ankle (HKA) angle was defined as the angle between the femoral and tibial mechanical axes. HKA angle is defined as a negative value in varus knee, whereas positive value in valgus knee. Mechanical lateral distal femoral angle (mLDFA) was defined as the lateral angle formed by the femoral mechanical axis and the distal femoral joint line. Joint line congruency angle (JLCA) was defined as the angle formed by distal femoral joint line and proximal tibial joint line. Medial convergence of JLCA was defined as a negative value, and a lateral JLCA was defined as a positive value. The medial proximal tibial angle (MPTA) was defined as the medial angle between the mechanical tibial axis and the proximal tibial joint line.

Measurement of bone cut simulation in frontal plane. The bone cut simulation was carried out at the request of the mechanical alignment (MA) technique using PACS. On the femoral side, a line (Line A) that both tangential to the most distal femur (either medial condyle or lateral condyle) and perpendicular to femoral mechanical axis was set. Then, another line (Line B) parallel to Line A and 9mm proximal to Line A was set. Finally, the distance from Line B to the most distal part of medial and lateral femoral condyles were measured and recorded as medial femoral resection thickness and lateral femoral resection thickness, respectively (Fig. 1a). On the tibial side, a line (Line C) that both tangential to the most prominent articular surface (either medial or lateral plateau) and perpendicular to tibial mechanical axis was set. Similarly, another line (Line $D$ ) parallel to Line $C$ and $10 \mathrm{~mm}$ distal to Line $C$ was set. The distance from Line $D$ to the two points on the medial and lateral concave aspect of the tibial plateau were measured as medial tibial resection thickness and lateral tibial resection thickness, respectively (Fig. 1b).

Estimate of total bone resection. The medial resection thickness was calculated as the sum of medial femoral resection thickness and medial tibial resection thickness. So was the lateral resection thickness. 
Regardless of the medial or lateral resection thickness, only the larger data (defined as "Max. resection" in the following text) enter the following calculation. Such algorithm is obligated under MA technique. In clinical scenario, in order to ensure the postoperative lower extremity alignment, distal femoral resection and proximal tibial resection must be perpendicular to the individual mechanical axis. As a result, a discrepancy between medial and lateral resection amount is very common. In case of the EG on one side is too narrow due to less bone is resected, ligament release is usually mandatory to achieve EG balance, rather than an extra parallel resection.

\section{Surgical procedure and intraoperative verification}

Operations were performed by two senior attending surgeons (ZW, LW). Gemini MK-II prostheses (Link, Hamburger, Germany) were used in this study, of which 131 were cruciate-retaining type and 6 were posterior stabilized type, and conventional instruments were used. The distal femoral resection was conducted perpendicular to the femoral mechanical axis (10), after the valgus resection angle was set accordingly, $9 \mathrm{~mm}$ of resection thickness was determined by the most prominent femoral condyle. Tibial resection was conducted perpendicular to the tibial mechanical axis, the resection level is estimated using the stylus, $10 \mathrm{~mm}$ from a less worn plateau or the most prominent side of lateral plateau. The EG was subsequently preliminary estimated. Measured resection technique were used in current study. When the bone preparation was completed, necessary ligament balancing were performed before the components were cemented.

Because this study is retrospective, we only collected the intraoperative resection data of the 10 patients finally included in this study for the verification of the accuracy of bone cut simulation. The resected bone pieces from distal femoral condyles and proximal tibia were calipered and recorded to compare with the preoperative radiographic measurement data $\mathbb{F i g} .2 \mathrm{a}, \mathrm{b} \bigotimes$. The magnification of radiograph is 1.05 .

\section{Collection of actually resection data retrospectively}

The original medical records of all included patients were retrospectively investigated. The patient's surgical records were reviewed: whether the patient received extra cut in distal femur or proximal tibia, whether a thicker liner was used, and whether an augment was used or applying thicker bone cement beneath the femoral component to balance the flexion-extension gap were collected for further analysis.

\section{Statistical analysis}

The data were summarized using means (SD) or medians. The normal distributions were tested by the Shapiro-Wilk test. Correlation analysis was used to investigate the relationship between Max. resection amount and various joint orientation parameters. A linear regression analysis was conducted to detect predictive factors for bone cut amount in extension. A reliability analysis using intraclass correlation coefficient (ICC) was conducted for intraoperative resection verification. All statistical tests were performed using SPSS statistical software (version 22; SPSS, Inc., Chicago, IL, USA). P value of $<0.05$ 
was considered statistically significant. All plots in this study were generated using R (4.0.0, R Core Team (2020), Vienna, Austria) and package ggplot2 (3.2.1, Wickham (2020), New York, US).

\section{Results}

Patients' demographics and various joint orientation parameters are shown in Table 1. Among the 137 knees included in this study, interestingly, the MPTAs in all varus knees are less than 90 degrees, and the mLDFAs in all valgus knees are less than 90 degrees. This finding could be understood as following: when MA technique was used in TKA for bone preparation, lateral tibial plateau resections are always thicker than medial ones in varus knees, while the resection thicknesses of medial femoral condyles are always greater than the ones of lateral condyles in valgus knees.

Varus knees can be divided into 2 subgroups based on the values of mLDFA: Subgroup 1, varus knee with mLDFA $<90^{\circ}$; and subgroup 2, varus knee with mLDFA $>90^{\circ}$. In the same way, valgus knee can be divided into 2 subgroup based on the values of MPTA: subgroup 3, valgus knee with MPTA $<90^{\circ}$, and subgroup 4, valgus knee with MPTA $>90^{\circ}$ (Table 1) (Fig. 3). Before further analysis, the reason for the above grouping is that in subgroup 2, the resection level of distal femur and tibial plateau refer to the lateral femoral condyle and lateral tibial plateau, respectively. After the resection, the lateral compartment has been acquired enough space to accommodate the components, and the medial compartment is usually narrower than the lateral compartment. Next, osteophyte removal and extensive soft tissue release are required to achieve EG balance. In subgroup 4, on the contrary, extensive lateral soft tissue release often needs to achieve EG balance after primary bone cut. Therefore, the data of subgroup 2 and subgroup 4 were not involved in the subsequent analysis because the Max. resection in both groups is constant. 
Table 1

Patients' demographics and radiographic joint line parameters

\begin{tabular}{|ll|}
\hline Number of patients & 112 \\
\hline Gender(male/female) & $25 / 87$ \\
\hline Age(years old, mean \pm SD) & $68.7 \pm 8.5$ \\
\hline Number of knees & 137 \\
\hline Side(left/right) & $67 / 70$ \\
\hline Deformity(varus/valgus) & $116 / 21$ \\
\hline HKA(degree, mean \pm SD) & $-5.6 \pm 6.2$ \\
\hline mLDFA(degree, mean \pm SD) & $88.1 \pm 2.9$ \\
\hline JLCA(degree, mean \pm SD) & $-3.6 \pm 3.1$ \\
\hline MPTA(degree, mean \pm SD) & $86.1 \pm 2.4$ \\
\hline Subgroup 1 (varus knee, mLDFA $<90^{\circ}$ ) & $84(61.3 \%)$ \\
\hline Subgroup 2 (varus knee, mLDFA $>90^{\circ}$ ) & $32(23.4 \%)$ \\
\hline Subgroup 3 (valgus knee, MPTA $<90^{\circ}$ ) & $14(10.2 \%)$ \\
\hline Subgroup 4 (valgus knee, MPTA $>90^{\circ}$ ) & $7(5.1 \%)$ \\
\hline
\end{tabular}

Radiographic measurement data of subgroup 1 and subgroup 3 were included for subsequent analysis. Key parameters, including HKA, mLDFA, JLCA, MTPA, and Max. resection, were tested for normality. The results showed that only mLDFA and Max. resection data in subgroup 1 conformed to the normal distribution, with $p=0.003$ and $p=0.004$, respectively.

With respect to the relationship of the resection amount and various joint orientation parameters, Spearman's correlation was conducted. The results showed that mLDFA and MPTA had a positive correlation with the Max. resection amount in subgroup 1, with correlation coefficient of 0.931 and 0.304 , with significance of $<0.001$ and 0.005 , respectively. Linear regression was subsequently conducted using mLDFA and MPTA to investigate which parameter had better predictive capacity. The regression equation was derived as follows: $\mathrm{y}=0.556 \mathrm{x}_{1}+0.098 \mathrm{x}_{2}-39.74$, where $\mathrm{y}$ represents Max. resection, $\mathrm{x}_{1}$ and $\mathrm{x}_{2}$ represent mLDFA and MPTA, respectively, $R^{2}=0.808$ (Fig. 4). On the other hand, Spearman's correlation showed that only MPTA have a weak positive correlation with the Max resection in subgroup 3, with correlation coefficient of 0.009 . The regression equation is $y=0.415 x-19.493, R^{2}=0.617$ (Fig. 5).

With respect to the intraoperative verification of the bone cut simulation, a reliability analysis was conducted. The intraclass correlation coefficient was 0.984 , with $95 \%$ confidence interval from 0.969 to 
0.991 , and $p<0.001$ in F test. Statistical analysis showed that the simulated bone resection in PACS had high consistency with the intraoperative measurement.

Retrospective surgical records showed that $70.8 \%$ of patients did not receive further adjustments (0) after the primary bone resection, while other patients received various intraoperative adjustments, including $2 \mathrm{~mm}$ of extra tibial cut ( $+2 \mathrm{t}), 2 \mathrm{~mm}$ of extra distal femoral cut $(+2 \mathrm{f})$, and both (+4), a $2 \mathrm{~mm}$ thicker liner $(-2 \mathrm{t})$, and very rarely, about $2 \mathrm{~mm}$ additional cement was applied between the distal femur and the component to deal with relatively wider EG (-2f). (Table 2.). No other types of adjustments were found in the review of the surgical records of current case series.

Table 2

Intraoperative adjustment of primary bone cut in different subgroups of patients

\begin{tabular}{|lllllll|}
\hline & $\mathbf{- 2 f}$ & $\mathbf{- 2 t}$ & $\mathbf{0}$ & $\mathbf{+ 2 f}$ & $\mathbf{+ 2 t}$ & $\mathbf{+ 4}$ \\
\hline Subgroup1 & 0 & 1 & 59 & 6 & 14 & 4 \\
\hline Subgroup2 & 0 & 4 & 24 & 1 & 1 & 2 \\
\hline Subgroup3 & 0 & 1 & 10 & 2 & 1 & 0 \\
\hline Subgroup4 & 2 & 0 & 4 & 0 & 1 & 0 \\
\hline Total(n, \%) & $2(1.5 \%)$ & $6(4.4 \%)$ & $97(70.8 \%)$ & $9(6.6 \%)$ & $17(12.4 \%)$ & $6(4.4 \%)$ \\
\hline
\end{tabular}

In order to investigate whether there was a certain correlation between intraoperative adjustment of primary resection and joint line orientation, grouped scatter plots are generated using surgical records' data from subgroup 1 and subgroup 3 . In subgroup 1, mLDFA is used as an independent variable because of its greater weight (Fig. 6a), while in group 3, MPTA was used as an independent variable (Fig. $6 \mathrm{~b}$ ). In the cases of varus knee with $\mathrm{mLDFA}<90^{\circ}$, as $\mathrm{mLDFA}$ decreases, the chances of bone recut will increase. No matter where the extra bone cut occurred, from femoral side or tibial side. Conversely, as mLDFA approaches $90^{\circ}$, the chances of using thicker liners slightly increase. On the other hand, in the case of valgus knee with MPTA $<90^{\circ}$, although MPTA and intraoperative adjustment of resection presents similar trends, this relationship is less persuasive because of fewer cases.

\section{Discussion}

In contrast with patients who receive primary total hip arthroplasty, approximately $20 \%$ of those who receive TKA are not satisfied with their outcomes $(11,12)$. Although its underlying reason is not clear, alignment technique of TKA is beginning to be considered to be related to the patient's postoperative outcomes and dissatisfaction $(13,14)$. With the application of more accurate alignment assistant instruments, the conventional MA technique has begun to be challenged in recent years $(7,15,16)$, because MA cannot fully respect the patient's natural alignment of lower extremities and joint line orientation, and a significant variation in overall coronal limb alignment and in femoral and tibial joint lines had been proved to exist in osteoarthritic knees(17). 
The safety and reliability of MA technique has been proved for decades $(1,18)$, so it is considered to be the "gold standard" for TKA $(19,20)$. This opinion is particularly important for developing countries. Taking China for example,because more accurate alignment-assistant instruments, such as navigation, patient-specific instrumentation (PSI) or robot assistant technology, can only be available in few hospitals $(21,22)$. In order to reduce the disastrous consequences caused by poor alignment after TKA, a more feasible and more reliable alignment method should be addressed when using conventional instruments for TKA. Therefore, the goal of this study was to investigate the correlation between the orientation of the joint line and the quantities of bone resection in TKA when MA and conventional instrument were used.

With respect to the classic MA philosophy, the distal femoral and proximal tibial resection should be perpendicular to the mechanical axis of femur and tibia, respectively. The resection thickness should be equal to corresponding components thickness to guarantee proper soft tissue tension in full range of motion without extra soft tissue release $(23,24)$. In most of patients, however, neither femoral condyles nor tibial plateau are perpendicular to the mechanical axis. As a consequence, the resection thicknesses of femoral condyles were not always the same, so was the tibial plateau(25).

In the femoral bone preparation, when a conventional instrument was used, a valgus alignment jig, together with an intramedullary rod, was usually applied to determine the orientation of the distal femoral cut. Theoretically, the distal femoral resection thickness was determined by adjustable jig, but actual resection thickness of each condyle was determined by which condyle contact with the jig first. In most of cases, the medial part of jig contacted the articular surface first, as a consequence, the resection depth of medial femoral condyle was usually equal to the desired thickness, while the resection thickness of lateral femoral condyle was usually less than that of medial condyle. On the other side, the resection thickness of medial tibial plateau was usually less than that of lateral plateau, especially in most of knee joint arthrosis with neutral or varus alignment. So, the amount of bone removed generally is less than that to be replaced by the components.

Although the discrepancy of medial and lateral EG could be corrected by osteophyte removal or ligament release, a gross narrower or wider EG would be caused by too little or too much bone removal. The reason for above clinical scenario is the combination of MA operative principles, surgeon's individual error and high variability of joint line orientation.

In one study (26), Hirschmann et al. have combined the individual HKA, femoral mechanical angle (supplementary angle of mLDFA) and tibial mechanical angle (same as MPTA) to divide the knee joint into a group of "functional knee phenotypes". The phenotypes were matched with the target of different TKA alignment techniques, and it was found that only $5.6 \%$ of the males and $3.6 \%$ of the females met the MA alignment target. In another study (27), Lin et al. investigated relative parameters of the lower limb alignment in relation to each other in a young non-osteoarthritic population, and all subjects were categorized on the basis of HKA, mLDFA and MPTA. In Lin's study, all subjects could be classified into one of five types of alignment: 2 types of neutral alignment, 2 types of varus alignment and 1 type of 
valgus alignment. The coronal neutral alignment in Hirschmann's study and Lin's study were defined as " $-1.5^{\circ}<H K A<11.5^{\circ}$ " and " $-3^{\circ}<H K A<3^{\circ}$ ", respectively, rather than HKA $=0^{\circ}$.

For the convenience of the evaluation of the correlation between the joint line orientation and the amount of bone resection in extension when MA technique was used, and in order to make the correlation more practicable in clinical situation, all subjects included in our study were patients diagnosed with knee OA, and all patients were divided into valgus knee group and varus knee group based on whether HKA is greater than $0^{\circ}$ or not. During the measurement of the alignment parameters, all varus knees were found to have MPTA less than $90^{\circ}$, and all valgus knees had mLDFA less than $90^{\circ}$. This finding is consistent with previous published literatures, the major contributors to valgus knee and varus knee are valgus of the distal femur(28) and varus of the proximal tibia(7), respectively.

In our case series, patients in varus knee group were divided into 2 subgroups based on if individual mLDFA is less than $90^{\circ}$ or not, while valgus knees were similarly divided with reference to MPTA. Usually, the coronal deformities in subgroup 2 and subgroup 4 are more serious than ones in other two subgroups, because both the femoral and the tibial bony variations contribute to the coronal deformity. Lateral bowing of femoral shaft may be an important reason leading to the relatively greater mLDFA in subgroup 2(29, 30). According to the MA technique requirement, bone resection should be perpendicular to the femoral and tibial mechanical axis. Therefore, when determining the level of bone resection, lateral condyle and lateral tibial plateau should be used as a reference in varus knees in subgroup 2. As a consequence, appropriate gap could be easily achieved in the lateral compartment, but extensive ligament release was inevitable in the medial compartment, because of the less bone removal and intrinsically tight medial collateral ligament (31). A similar situation would occur in valgus knees in subgroup 4, in which the bone resection levels are determined by medial condyle and medial tibial plateau. Extensive soft tissue release or even constrained prostheses would be used for achieving acceptable mediolateral balance. Hence, only the data of simulated resection in subgroup 1 and subgroup 3 were used for further analysis.

As far as we know, no study focusing on the relationship between the joint line orientation and the amount of bone resection has been performed before. One study found a significant correlation between leg axis and the optimal tibial resection thickness, and the optimal resection thickness in valgus knees were significantly less than that in neutral or varus knees, but no joint line orientation parameters was investigated (8). Inconsistent with the results of the above study, the amount of bone resection was not related to HKA in current study. The reason for this inconsistent result may be due to the fact that only joint line orientation parameters and simulated resection data of subgroup 1 and subgroup 3 were included for correlation analysis.

Patients in subgroup 1 have varus knees with valgus femurs, and account for the majority of all included patients (61.3\%). Correlation analysis showed that both MPTA and mLDFA are positively related to the amount of bone resection. The subsequent regression analysis results showed that mLDFA rather than MPTA can have a significant effect on the amount of bone resection, with regression coefficient of 0.556 
and 0.098 , respectively. This was truly an unexpected finding. The interpretation of the results of regression analysis showed that for every $2^{\circ}$ increase in mLDFA, the bone cut amount will increase by about $1 \mathrm{~mm}$. Given joint line inclination of $3^{\circ}$ of varus as average value, when mLDFA is approaching $90^{\circ}$, the EG will increase by $1.5 \mathrm{~mm}$, and when $\mathrm{mLDFA}$ is $85^{\circ}$, the EG will decrease by $1.5 \mathrm{~mm}$. For MPTA, although there is a certain degree of correlation with bone resection quantity, its impact is so weak that can even be ignored.

Patients in subgroup 3 have valgus knees with varus tibia, and account for the majority of the valgus knees. Only MPTA had been proven to be related with the bone resection amount. In contrast with mLDFA in subgroup 1, MPTA has a weaker influence on the bone resection, both the regression coefficient and $\mathrm{R}^{2}$ are smaller than mLDFA in subgroup 1. The small number of patients in subgroup 3 might be part of the reason.

The effectiveness of simulated bone cut has been verified by intraoperative calipered bone resection in this study. The retrospective medical records queries did show that the actual bone resection adjustment during surgery had a certain correlation with the corresponding joint line parameters. Although data visualization can show us some useful information, we also found that not all bone resection adjustments occurred in subgroup 1 and subgroup3, this phenomenon presented that the operation error still has a significant impact on the accuracy of bone resection.

Our study has several limitations. First, a small number of patients were included in our study. And there may be some selection bias as we excluded some patients due to rotation of lower extremities in fulllength weight bearing radiogram (32). In addition, because of the limited sample size, it may be that not all patients diagnosed with knee OA could be classified into 4 subgroups. One study reported that a valgus knee with mLDFA as large as $92^{\circ}$ (33). Secondly, some special type of extra-articular deformities, such as tibial or femoral shaft bowing with the progression of knee OA, were not used as alignment parameters. Although tibial or femoral bowing could change the joint line orientation dramatically, such deformities rarely need correction during primary TKA, so our study did not focus on these special extraarticular deformities. Finally, full-length weight bearing radiographs rather than 3D CT were used for radiographic measurement, and some study have proven that the latter has higher accuracy (34). Actually, the cost effectiveness of radiograph is much better. As a routine before TKA, some meaningful finding in preoperative radiographs would be more helpful for guiding clinical practice.

\section{Conclusion}

Knee joint deformities in patients diagnosed with OA could be artificially divided into 4 subgroups based on the alignment of extremities and the joint line orientations. Varus knee with $m L D F A<90^{\circ}$, varus knee with $\mathrm{mLDFA}>90^{\circ}$, valgus knee with MPTA $<90^{\circ}$ and valgus knee with MPTA $>90^{\circ}$. Patients in subgroup 1 account for majority of all patients $(61.3 \%)$ in current case series. When a TKA is performed in varus knee with $\mathrm{mLDFA}<90^{\circ}$ using conventional instrument and MA technique, the amount of bone resection in EG 
is linearly related to the patient's mLDFA, every $2^{\circ}$ of increase in mLDFA, the amount of bone resection will increase by about $1 \mathrm{~mm}$.

\section{Abbreviations}

TKA: Total knee arthroplasty; MA: Mechanical alignment; OA: Osteoarthritis; EG: Extension gap; AP: Anterioposterior; PACS: Picture archiving and communication systems; HKA: Hip-knee-ankle; mLDFA: Mechanical lateral distal femoral angle; JLCA: Joint line congruency angle; MPTA: Medial proximal tibial angle

\section{Declarations}

\section{Acknowledgements}

No other acknowledgements applicable

\section{Declarations of interest}

None

\section{Funding}

National Natural Science Foundation of China (81572180)

\section{Availability of data and materials}

The raw data used and analyzed in the current study are available on reasonable request. Please contact first author or corresponding author for data requests.

\section{Author's contributions}

ZWW contributed to the study design, performed the surgical procedure, statistical interpretation and drafted the manuscript. YY performed radiographic measurement, collected raw data and retrospective review patients' medical records. LW performed the surgical procedures, intraoperative bone resection measurement and data analysis. DSM collected the intraoperative measurement data and participated data collation and analysis. TBQ contributed to the study design, gained ethics approval and clinical coordination. All authors have read and approved the final manuscript. 


\section{Ethics approval and consent to participate}

This study was approved by the Ethic board of Beijing Chaoyang Hospital, National Natural Science Foundation of China. Surgical records' data was recorded retrospectively and no extra individual consent was needed to participate in this study.

\section{Consent for publication}

Not applicable

\section{Competing interests}

The authors declare that they have no competing interests.

\section{References}

1. Fang DM, Ritter MA, Davis KE. Coronal alignment in total knee arthroplasty: just how important is it? J Arthroplast. 2009;24(6 Suppl):39-43.

2. Lombardi AV Jr, Berend KR, Ng VY. Neutral mechanical alignment: a requirement for successful TKA: affirms. Orthopedics. 2011;34(9):e504-6.

3. Ritter MA, Davis KE, Meding JB, Pierson JL, Berend ME, Malinzak RA. The effect of alignment and $\mathrm{BMI}$ on failure of total knee replacement. The Journal of bone joint surgery American volume. 2011;93(17):1588-96.

4. Berend ME, Ritter MA, Meding JB, Faris PM, Keating EM, Redelman R, et al. The Chetranjan Ranawat Award: Tibial Component Failure Mechanisms in Total Knee Arthroplasty. Clin Orthop Relat Res. 2004;428:26-34.

5. Green GV, Berend KR, Berend ME, Glisson RR, Vail TP. The effects of varus tibial alignment on proximal tibial surface strain in total knee arthroplasty: The posteromedial hot spot. J Arthroplast. 2002;17(8):1033-9.

6. Victor JM, Bassens D, Bellemans J, Gursu S, Dhollander AA, Verdonk PC. Constitutional varus does not affect joint line orientation in the coronal plane. Clin Orthop Relat Res. 2014;472(1):98-104.

7. Bellemans J, Colyn W, Vandenneucker H, Victor J. The Chitranjan Ranawat award: is neutral mechanical alignment normal for all patients? The concept of constitutional varus. Clin Orthop Relat Res. 2012;470(1):45-53.

8. Schnurr C, Csécsei G, Nessler J, Eysel P, König DP. How much tibial resection is required in total knee arthroplasty? International orthopaedics. 2010;35(7):989-94. 
9. Paley D, Herzenberg JE, Tetsworth K, McKie J, Bhave A. Deformity planning for frontal and sagittal plane corrective osteotomies. The Orthopedic clinics of North America. 1994;25(3):425-65.

10. Tan $H$, Wang $Y$, Long T, Nie B, Mao Z, Yue B. How to accurately determine the distal femoral valgus cut angle in the valgus knee arthroplasty. International orthopaedics. 2018;42(3):537-42.

11. Bourne RB, Chesworth BM, Davis AM, Mahomed NN, Charron KD. Patient satisfaction after total knee arthroplasty: who is satisfied and who is not? Clin Orthop Relat Res. 2010;468(1):57-63.

12. Robertsson O, Dunbar M, Pehrsson T, Knutson K, Lidgren L. Patient satisfaction after knee arthroplasty: a report on 27,372 knees operated on between 1981 and 1995 in Sweden. Acta Orthop Scand. 2000;71(3):262-7.

13. Lording T, Lustig S, Neyret P. Coronal alignment after total knee arthroplasty. EFORT open reviews. 2016;1(1):12-7.

14. An VVG, Twiggs J, Leie M, Fritsch BA. Kinematic alignment is bone and soft tissue preserving compared to mechanical alignment in total knee arthroplasty. Knee. 2019;26(2):466-76.

15. Rivière $C$, Iranpour F, Auvinet E, Aframian A, Asare K, Harris S, et al. Mechanical alignment technique for TKA: Are there intrinsic technical limitations? Orthopaedics \& Traumatology: Surgery \& Research. 2017;103(7):1057-67.

16. Allen MM, Pagnano MW. Neutral mechanical alignment: Is it Necessary? The bone \& joint journal. 2016;98-B(1 Suppl A):81 - 3.

17. Hess S, Moser LB, Amsler F, Behrend H, Hirschmann MT. Highly variable coronal tibial and femoral alignment in osteoarthritic knees: a systematic review. Knee surgery, sports traumatology, arthroscopy: official journal of the ESSKA. 2019;27(5):1368-77.

18. Lombardi AV, Berend KR, Ng VY. Neutral mechanical alignment: a requirement for successful TKA: affirms. Orthopedics. 2011;32(9):691-.

19. Schiraldi M, Bonzanini G, Chirillo D, de Tullio V. Mechanical and kinematic alignment in total knee arthroplasty. Annals of translational medicine. 2016;4(7):130.

20. Abdel MP, Oussedik S, Parratte S, Lustig S, Haddad FS. Coronal alignment in total knee replacement: historical review, contemporary analysis, and future direction. The bone joint journal. 2014;96$\mathrm{B}(7): 857-62$.

21. Wang ZW, Wen L, Yu Y, Ma DS, Zhou L, Zhang B, et al. [Early clinical effects of total knee arthroplasty with kinematic alignment assisted by patient-specific instrumentation]. Zhonghua wai ke za zhi [Chinese journal of surgery]. 2020;58(6):457-63.

22. Shen Z, Wang H, Duan Y, Wang J, Wang F. Application of 3D printed osteotomy guide plate-assisted total knee arthroplasty in treatment of valgus knee deformity. J Orthop Surg Res. 2019;14(1):327.

23. Rand J. Preoperative planning for total knee arthroplasty. Orthopedic knowledge update Hip and knee reconstruction. Rosemont: American Academy of Orthopedic Surgeons; 1995.

24. Yoshii I, Whiteside LA, White SE, Milliano MT. Influence of prosthetic joint line position on knee kinematics and patellar position. J Arthroplast. 1991;6(2):169-77. 
25. Zeng YM, Wang Y, Zhu ZA, Dai KR. Effects of sex and lower extremity alignment on orientation of the knee joint line in knee surgery. Chin Med J. 2012;125(12):2126-31.

26. Hirschmann MT, Moser LB, Amsler F, Behrend H, Leclerq V, Hess S. Functional knee phenotypes: a novel classification for phenotyping the coronal lower limb alignment based on the native alignment in young non-osteoarthritic patients. Knee surgery, sports traumatology, arthroscopy: official journal of the ESSKA. 2019;27(5):1394-402.

27. Lin YH, Chang FS, Chen KH, Huang KC, Su KC. Mismatch between femur and tibia coronal alignment in the knee joint: classification of five lower limb types according to femoral and tibial mechanical alignment. BMC Musculoskelet Disord. 2018;19(1):411.

28. Rossi R, Rosso F, Cottino U, Dettoni F, Bonasia DE, Bruzzone M. Total knee arthroplasty in the valgus knee. International orthopaedics. 2014;38(2):273-83.

29. Matsumoto T, Hashimura M, Takayama K, Ishida K, Kawakami Y, Matsuzaki T, et al. A radiographic analysis of alignment of the lower extremities-initiation and progression of varus-type knee osteoarthritis. Osteoarthritis cartilage. 2015;23(2):217-23.

30. Kim JM, Hong SH, Kim JM, Lee BS, Kim DE, Kim KA, et al. Femoral shaft bowing in the coronal plane has more significant effect on the coronal alignment of TKA than proximal or distal variations of femoral shape. Knee surgery, sports traumatology, arthroscopy: official journal of the ESSKA. 2015;23(7):1936-42.

31. Bellemans J, Vandenneucker H, Vanlauwe J, Victor J. The influence of coronal plane deformity on mediolateral ligament status: an observational study in varus knees. Knee surgery, sports traumatology, arthroscopy: official journal of the ESSKA. 2010;18(2):152-6.

32. Jamali AA, Meehan JP, Moroski NM, Anderson MJ, Lamba R, Parise C. Do small changes in rotation affect measurements of lower extremity limb alignment? J Orthop Surg Res. 2017;12(1):77.

33. Eberbach H, Mehl J, Feucht MJ, Bode G, Sudkamp NP, Niemeyer P. Geometry of the Valgus Knee: Contradicting the Dogma of a Femoral-Based Deformity. Am J Sports Med. 2017;45(4):909-14.

34. Eckhoff DG, Bach JM, Spitzer VM, Reinig KD, Bagur MM, Baldini TH, et al. Three-dimensional mechanics, kinematics, and morphology of the knee viewed in virtual reality. The Journal of bone joint surgery American volume. 2005;87(Suppl 2):71-80.

\section{Figures}



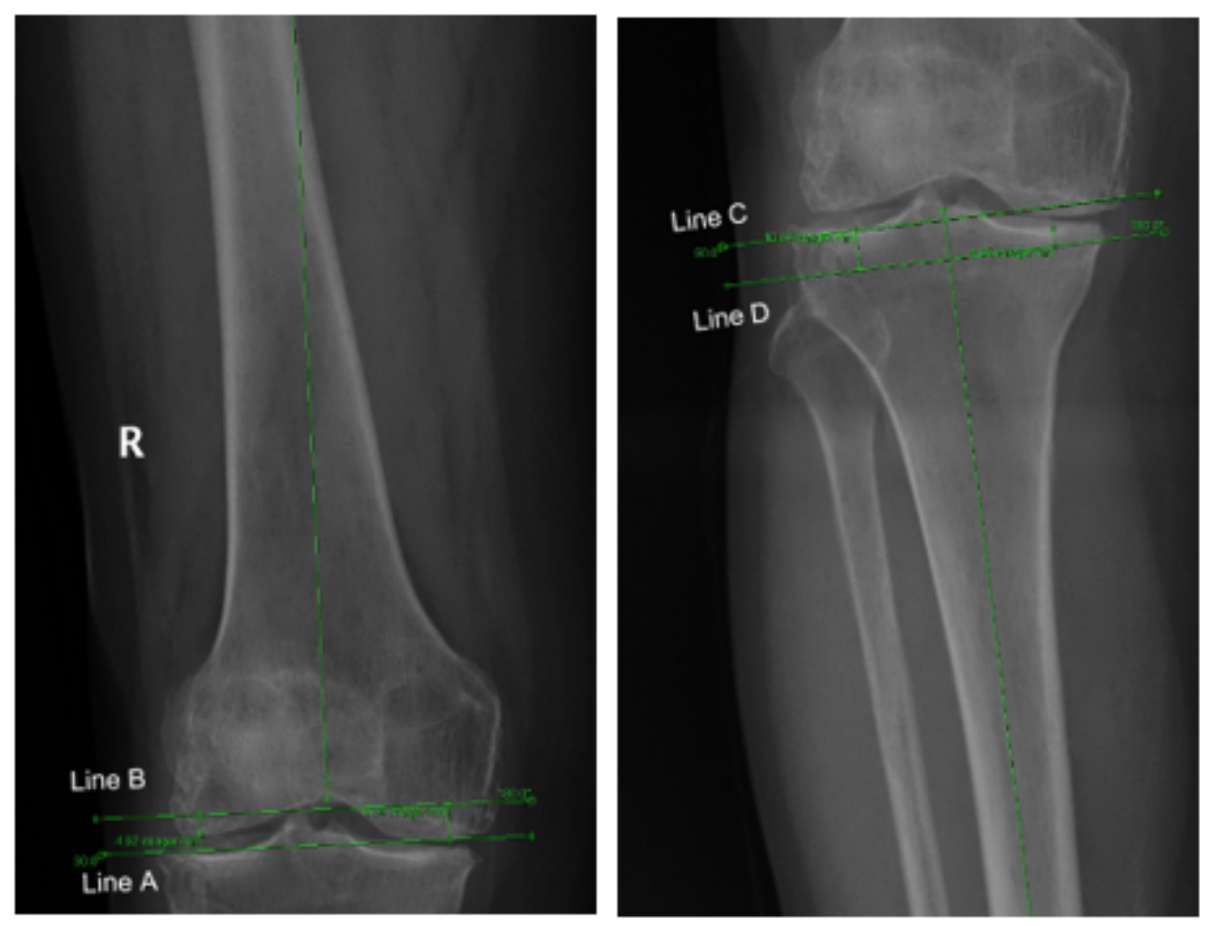

\section{Figure 1}

Simulated resection were performed in full-length weight bearing radiograph. The thickness of resection of the medial and lateral femoral condyles (a) and tibial plateau (b) were measured and recorded.
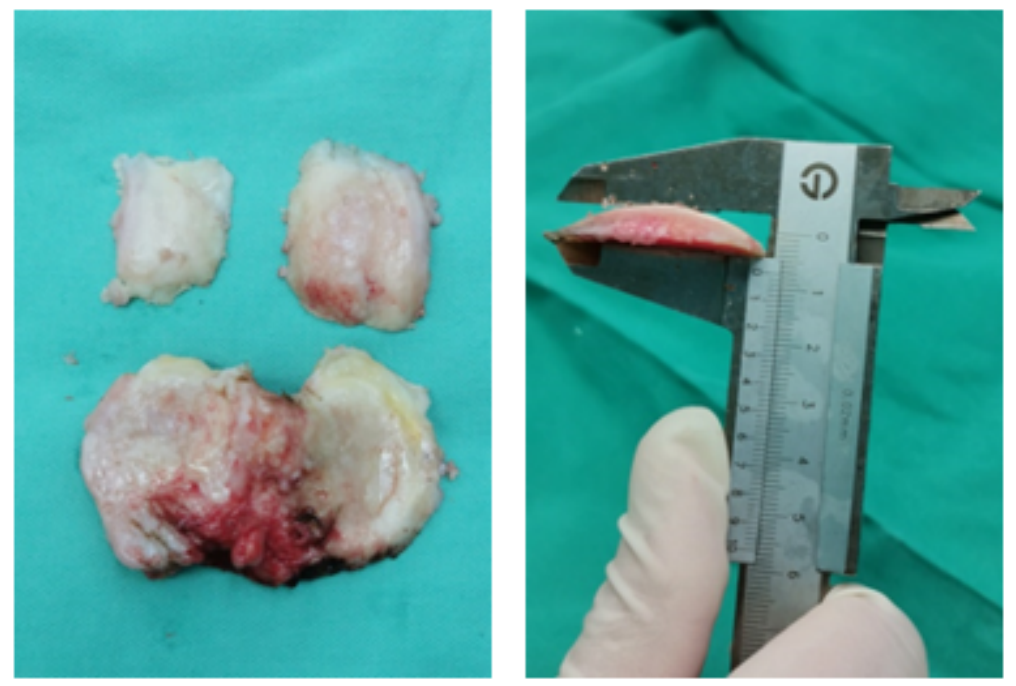

\section{Figure 2}

Demonstrated the resected tibial plateau and the distal femoral condyles in TKA (a). A caliper measured the thickness of the distal medial femoral condyle (b). In case of cartilage wear, oscillating saw kerf $(1.5 \mathrm{~mm})$ should be compensated. 

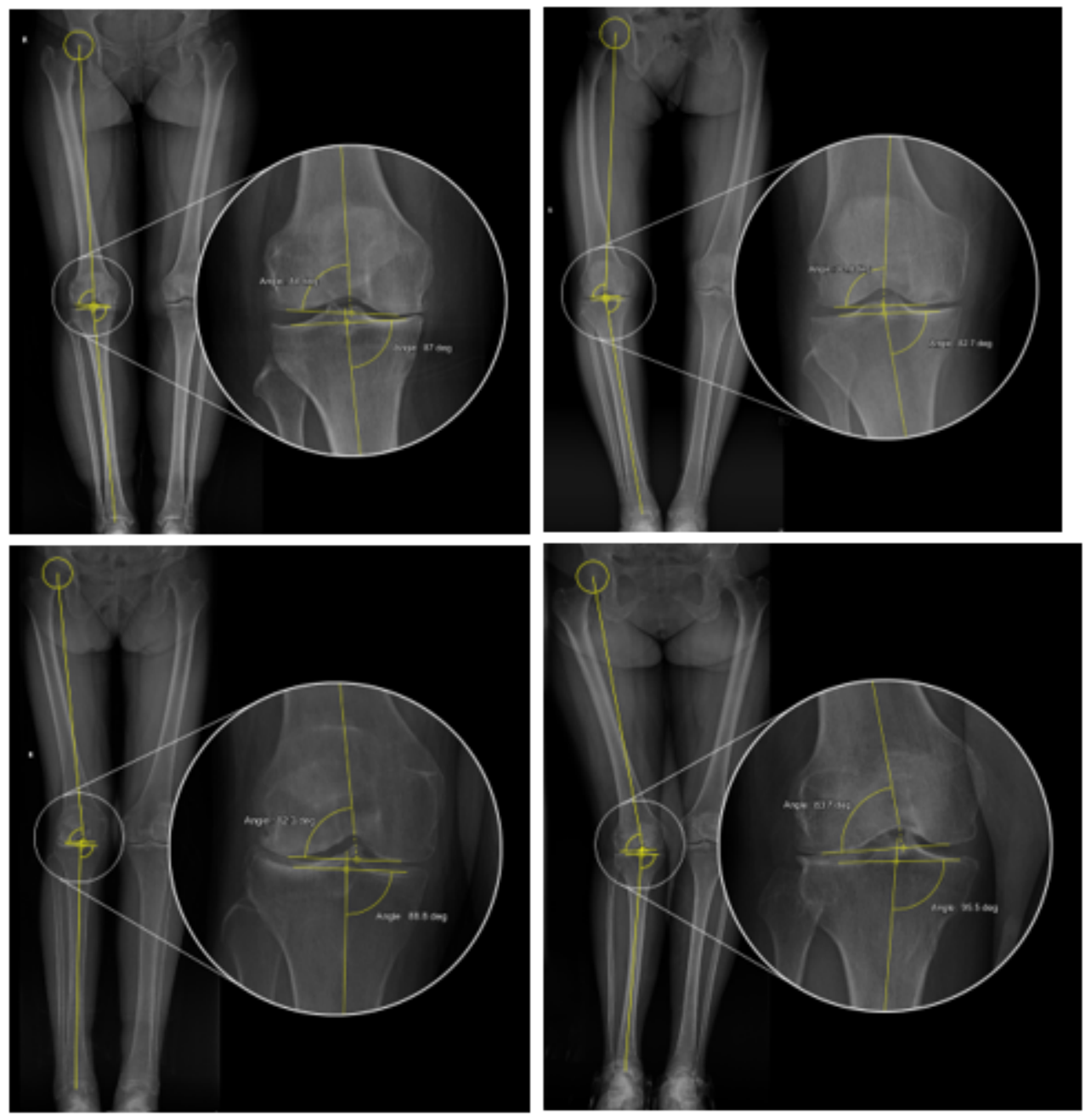

Figure 3

Patients can be divided into 4 subgroups based on limb alignment and joint orientation: (a) varus knee with $\mathrm{mLDFA}<90^{\circ}$, (b) varus knee with $\mathrm{mLDFA}>90^{\circ}$, (c) valgus knee with MPTA $<90^{\circ}$ and (d) valgus knee with MPTA $>90^{\circ}$.

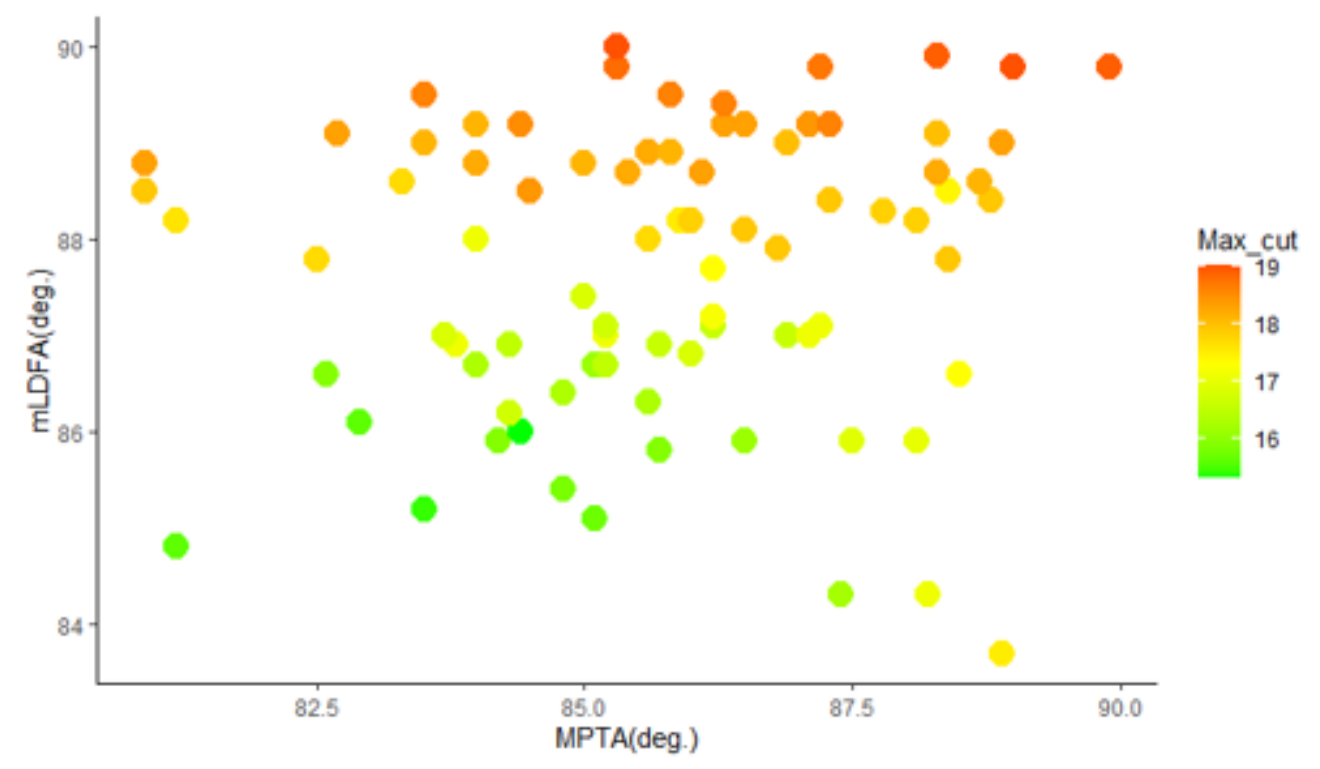




\section{Figure 4}

The relationship between Max. resection and joint orientation parameters in varus knee with Mldfa $<90$ degrees. Scatter dots weighted by Max. resection are shown. Compared with less bone cuts (green dots) in the bottom left quadrant, more bone cuts (red dots) appear in the upper quadrant rather than the most right quadrant. This shows that mLDFA has more impact on the amount of bone cut.

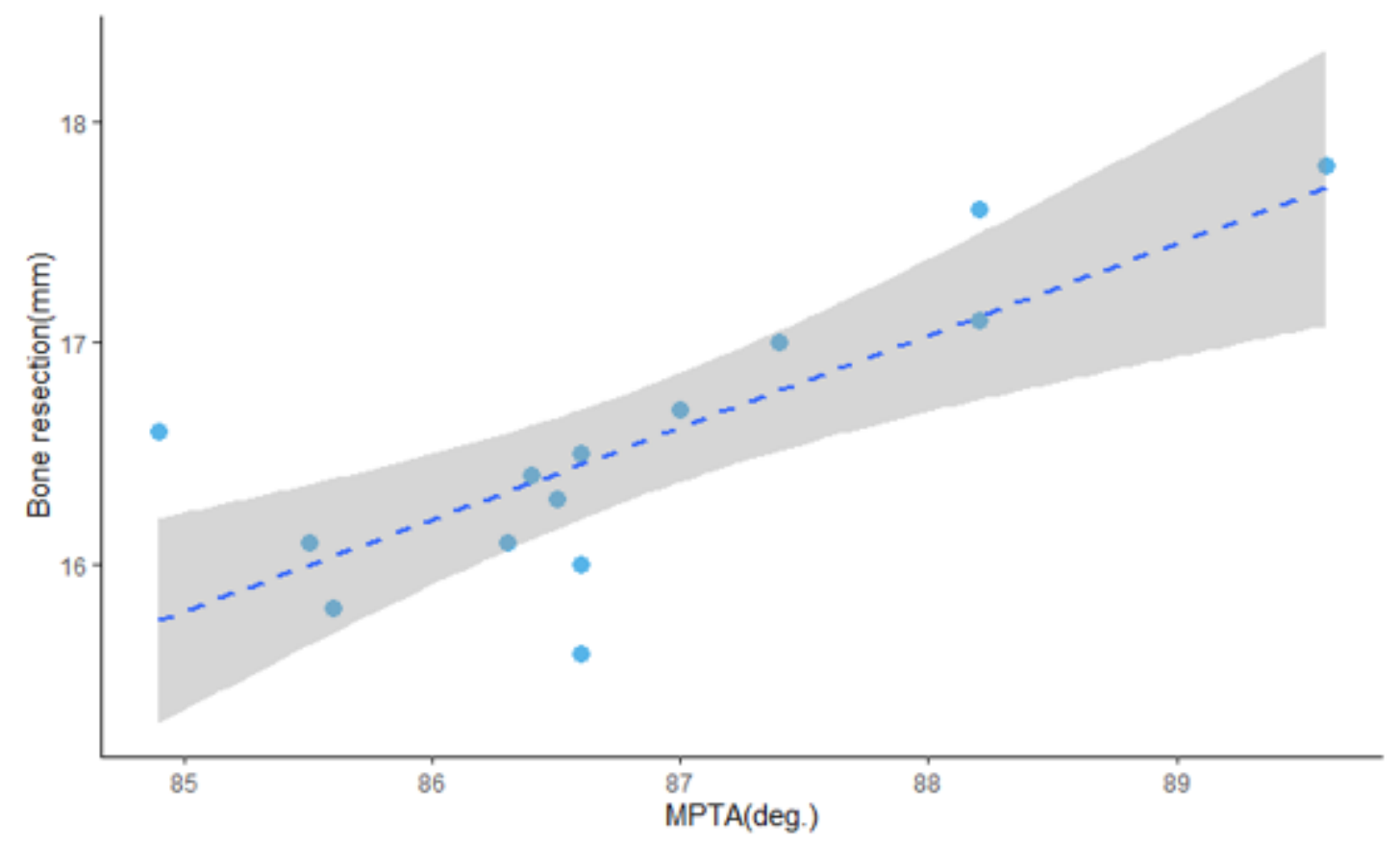

\section{Figure 5}

Linear regression plot of Max. resection and MPTA in valgus knee with mLDFA $<90$ degrees. The shaded part in the plot is the $95 \%$ confidence interval. 

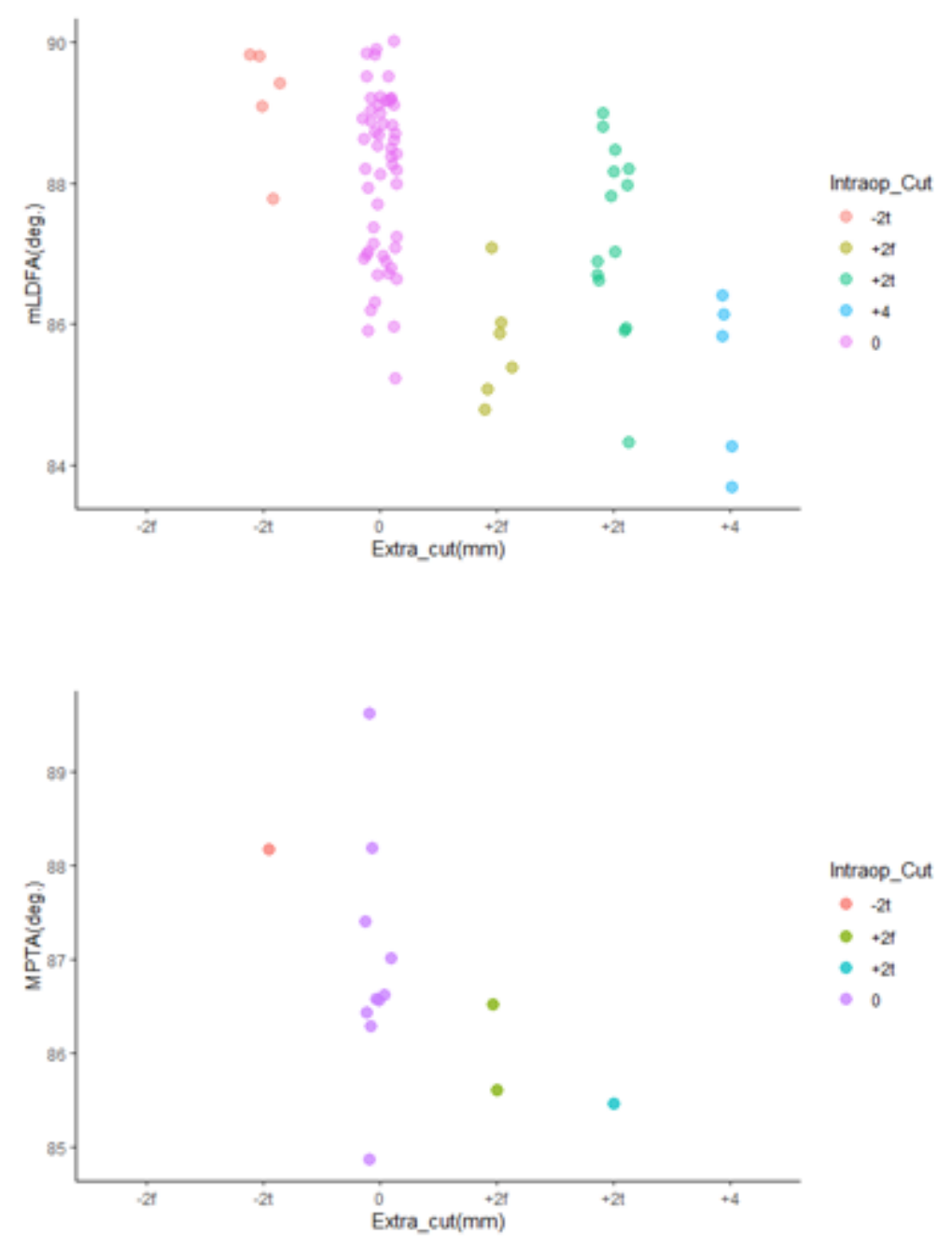

Figure 6

The relationship between intraoperative adjustment of bone cut and mLDFA in subgroup 1 (a), and MPTA in subgroup 3 (b). Note: 0 no bone cut adjustment, $+2 \mathrm{t} 2 \mathrm{~mm}$ of tibial recut, $+2 \mathrm{f} 2 \mathrm{~mm}$ of distal femoral recut, $+42 \mathrm{~mm}$ of tibial and $2 \mathrm{~mm}$ of femoral recut, $-2 \mathrm{t}$ applied $2 \mathrm{~mm}$ thicker liner. 